\title{
Prevalence of Metabolic Syndrome and Its Components among Saudi Young Adults 18 - 30 Years of Age
}

\author{
Abdalla A. Saeed \\ Community Medicine Faculty of Medicine, King Fahad Medical City, Riyadh, KSA \\ Email: saeed.abdalla@gmail.com, aasaeed@kfmc.med.sa
}

How to cite this paper: Saeed, A.A. (2019) Prevalence of Metabolic Syndrome and Its Components among Saudi Young Adults 18 - 30 Years of Age. Open Journal of Endocrine and Metabolic Diseases, 9, 49-59. https://doi.org/10.4236/ojemd.2019.94005

Received: February 20, 2019

Accepted: April 21, 2019

Published: April 24, 2019

Copyright () 2019 by author(s) and Scientific Research Publishing Inc. This work is licensed under the Creative Commons Attribution International License (CC BY 4.0).

http://creativecommons.org/licenses/by/4.0/

\section{(c) (i) Open Access}

\begin{abstract}
Background: Metabolic syndrome (MS) is a cluster of established cardiovascular risk factors that collectively increase predisposition to major chronic diseases, including heart diseases and diabetes mellitus. Young adults can be affected by MS or any of its components which predispose them to its complications later in their life. Hence early detection of MS and its components can be of help in preventing or controlling its adverse consequences among young adults. Materials and Methods: A total of 1354 Saudis aged 18 - 30 was randomly selected from 20 regions in the Kingdom of Saudi Arabia (KSA). Anthropometrics were collected, and fasting blood samples were collected to ascertain fasting blood glucose and lipid profile. Components of full MS and its components according to the International Diabetes Federation (IDF) were used in this study. Results: Metabolic syndrome prevalence was $12 \%$ and was significantly higher among males, advancing age and government employees and unemployed subjects but not according to income or education levels MS was significantly higher among current smokers, subjects with low physical activity level, but not significant according to dietary habits in terms of combined fruits and vegetables consumption. Low levels of High Density Lipoprotein (HDL) were the most frequent component in both males and females. Both waist circumference (WC) and low levels of HDL were much higher among females. Significant predictors included male gender, advancing age, lower level of physical activity and current smoking. Conclusion: The prevalence of MS is $12 \%$ in young adults. Low HDL is the most prevalent component of MS in young adults and thus may also be the first detectable component of MS in many young adults. Early identification of MS components could lead to targeted interventions to prevent the development of the syndrome, and thus reduce cardiovascular disease risk in later life.
\end{abstract}




\section{Keywords}

Metabolic Syndrome, Young Adults, Saudi Arabia

\section{Introduction}

Metabolic Syndrome (MS) is morbidity asymptomatic initially with features of hypertension, dyslipidemia, obesity, insulin resistance and dysglycemia. The person is diagnosed as having MS depends on the definition used which usually includes at least three of the following five components: large waist circumference, elevated triglycerides, low HDL, raised blood pressure, and elevated fasting blood Glucose. Hence the prevalence of MS will depend on which of these components are used and other factors such as demographics, life style habits and other factors [1] [2] [3] [4]. Investigating MS and its components among young adults is a worthwhile project as even the presence of just a single component increases the risk of future MS and cardiovascular morbidity in young adults [5] [6]. Hence early detection of any component of MS will be very helpful in intervention strategies with significant public health benefit. Several studies were conducted worldwide addressing MS using recommended methodology the IDF definition of MS [2] [7].

\section{Materials and Methods}

This was a cross-sectional, community-based study that covered the entire population of KSA in 2005. The WHO STEP wise approach to surveillance (STEPS) of noncommunicable diseases (NCD) risk factors was the basis of the conduct of the survey and collection of the data [2] [8] [9]. The STEPS approach focuses on obtaining core data on the established risk factors that determine the major disease burden. The STEPS instrument covers three different levels of "steps" of risk factor assessment. They are: Questionnaire, Physical measurements and Biochemical measurements. The original survey covers all Saudi nationals aged 15 - 64 years from all the 20 health regions of the country made up the study population. Stratified cluster random sampling technique was used to recruit the subjects. Stratification was based on age (five 10-year age groups) and gender (male/female, two groups). All health regions of the country (20 regions) were covered. Based upon the proposed methodology of the WHO STEP wise approach, a sample size of 196 was calculated for each of these categories. A list of all primary health care centers (PHCCs) in each region was prepared; $10 \%$ of these PHCCs were randomly chosen and allocated a regional sample proportionate to the size of their catchment population in the sampled PHCCs. To identify the households, a map of the health center coverage area was used to select the houses. Each house was assigned a number, and a simple random draw was made. Data were collected using the WHO STEP wise approach, a tool used for 
epidemiologic studies to measure NCD in WHO-member countries [8] [9]. It covers three levels of risk factor assessment (steps) which include a questionnaire, physical (anthropometric) and biochemical measurements including fasting serum glucose, lipid profile (triglycerides (TGs), total, HDL-C and low-density lipoprotein (LD)), chronic diseases (noncommunicable e.g. hypertension, dyslipidemia, diabetes mellitus, etc.), and risk factors (e.g., obesity, smoking, physical activity, diet). The questionnaire was translated into Arabic by a team of physicians and then translated back to ensure its accuracy. The Arabic instrument was pretested and corrected before being tested on 51 eligible respondents to check the wording and the clarity of the questions. Necessary adjustments were made to the instrument in the light of the pretest. Data was collected by 54 male and 54 female data collectors working in teams. Each field team was made up of four persons: A male data collector, a female data collector, a driver, and a female assistant. The data collection teams were supervised by a hierarchy of a local supervisor, regional coordinators, and national coordinator. All individuals involved in data collection attended a comprehensive training workshop of interview techniques, data collection tools, practical applications, and field guidelines. Blood $(5 \mathrm{ml})$ was collected, from the participants in the morning after an overnight fast. Sodium heparin was used as an anticoagulant, and the samples were centrifuged at $3000 \times \mathrm{g}$ for $15 \mathrm{~min}$ at $20^{\circ} \mathrm{C}$ to separate plasma. Aliquots were prepared for storage $\left(-20^{\circ} \mathrm{C}\right.$ or $\left.-80^{\circ} \mathrm{C}\right)$ until further analysis. Total cholesterol (TC), TGs, and glucose were measured with commercially available enzymatic colorimetric kits from QCA (Amposta, Spain). Seriscann normal (ref 994148) (QCA, Amposta, Spain) was used for quality control. Serum HDL levels were analyzed by an enzymatic method after precipitating serum reagents with phosphotungstic acid and magnesium. LDL-C was calculated according to the Friedewald formula (LDL-C = TC - HDL - [TG/5]). Height, weight, and waist and hip circumferences were measured using standard instruments, according to the STEP wise approach [8] [9] Body weight and height were measured without shoes, using electronic measuring scale. Body mass index (BMI) was calculated as weight in kilogram divided by height in $\mathrm{m}^{2}$. WC was measured, in $\mathrm{cm}$, midway between the lower costal margin and iliac crest during the end-expiratory phase. For the definition of MS, the IDF for MS was used in this study as follows [7]. Central obesity (defined as WC $\geq 94 \mathrm{~cm}$ for Europid men and $\geq 80 \mathrm{~cm}$ for Europid women, with ethnicity specific values for other groups). Plus, any two of the following four factors:

1) Raised TG level: $\geq 150 \mathrm{mg} / \mathrm{dL}(1.7 \mathrm{mmol} / \mathrm{L})$,

2) Reduced HDL: $<40 \mathrm{mg} / \mathrm{dL}(1.03 \mathrm{mmol} / \mathrm{L})$ in males and $<50 \mathrm{mg} / \mathrm{dL}(1.29$ $\mathrm{mmol} / \mathrm{L}$ ) in females,

3) Raised blood pressure (BP): Systolic Blood Pressure (SBP) $\geq 130$ or Diastolic Blood Pressure (DBP) $\geq 85 \mathrm{~mm} \mathrm{Hg}$,

4) Raised fasting plasma glucose $\geq 100 \mathrm{mg} / \mathrm{Dl}$ ( $5.6 \mathrm{mmol} / \mathrm{L})$.

The case definition of MS was generated using statistical analysis system sta- 
tistical package.

Questionnaires collected from the field were reviewed by the team leaders assigned to each team before submission to headquarters for data entry. A double entry of the questionnaires was done using Epi-Info 2000 software and EpiData software developed by the Menzes center for validation. After entry, the data, was cleaned. New variables were defined by adopting the standard STEPS variables (STEPS data management manual, draft version v1.5, October 2003). Collected data were cleaned, entered and statistically analyzed using SPSS for Windows, version 17.0 (Chicago, IL, USA). The data were given as mean and standard deviation for continuous variables and as frequencies (percentages) for categorical variables. Association between categorical variables was assessed using a Chi-square test. Differences between means were checked using t test or ANOVA or Manwhitnney or Kruskall tests as appropriate after checking for normality. Logistic regression analysis was used to identify predictors of MS and its individual components the level of significance was set at $<0.05$ throughout the study. Total counts may vary because of missing data from certain variables. The protocol and the survey instrument were approved by the ministry of health, center of biomedical ethics, and the appropriate authorities in KSA. The Institute Review Board (IRB) of King Fahad Medical City approved this study (IRB 19-090 dated 12 February 2019). Informed consent of all subjects was obtained. Participants were assured of confidentiality of data, and that they would be used only for the stated purpose of the survey. For this study only all young adults aged 18 - 30 years of age were selected from all subjects 15 - 64 years of age included in the survey.

\section{Results}

The young subjects aged 18 - 30 years were 1514 and 160 (10.6\%) of them were excluded from final analysis due to missing data and there were no significant differences according to demographic characteristics between these 160 excluded subjects and the 1354 included subjects. Table 1 shows their demographic characteristics and prevalence of MS among the 1354 subjects included in the analysis. MS prevalence was $12 \%$ and was significantly higher among males, advancing age and government employees and unemployed subjects but not according to income or education levels.

Table 2 profiles MS according to some life style characteristics. Metabolic syndrome was significantly higher among current smokers, subjects with low physical activity level, but not significant according dietary habits in terms of combined fruits and vegetables consumption.

Table 3 depicts the frequency of each of the five components of MS among the study subjects. HDL was the most frequent component in both males and females. Both WC and low levels of HDL were much higher among females.

There were no significant differences in TGs, fasting blood glucose and hypertension according to demographic characteristics of subjects except age 
Table 1. Metabolic syndrome according to demographic characteristics.

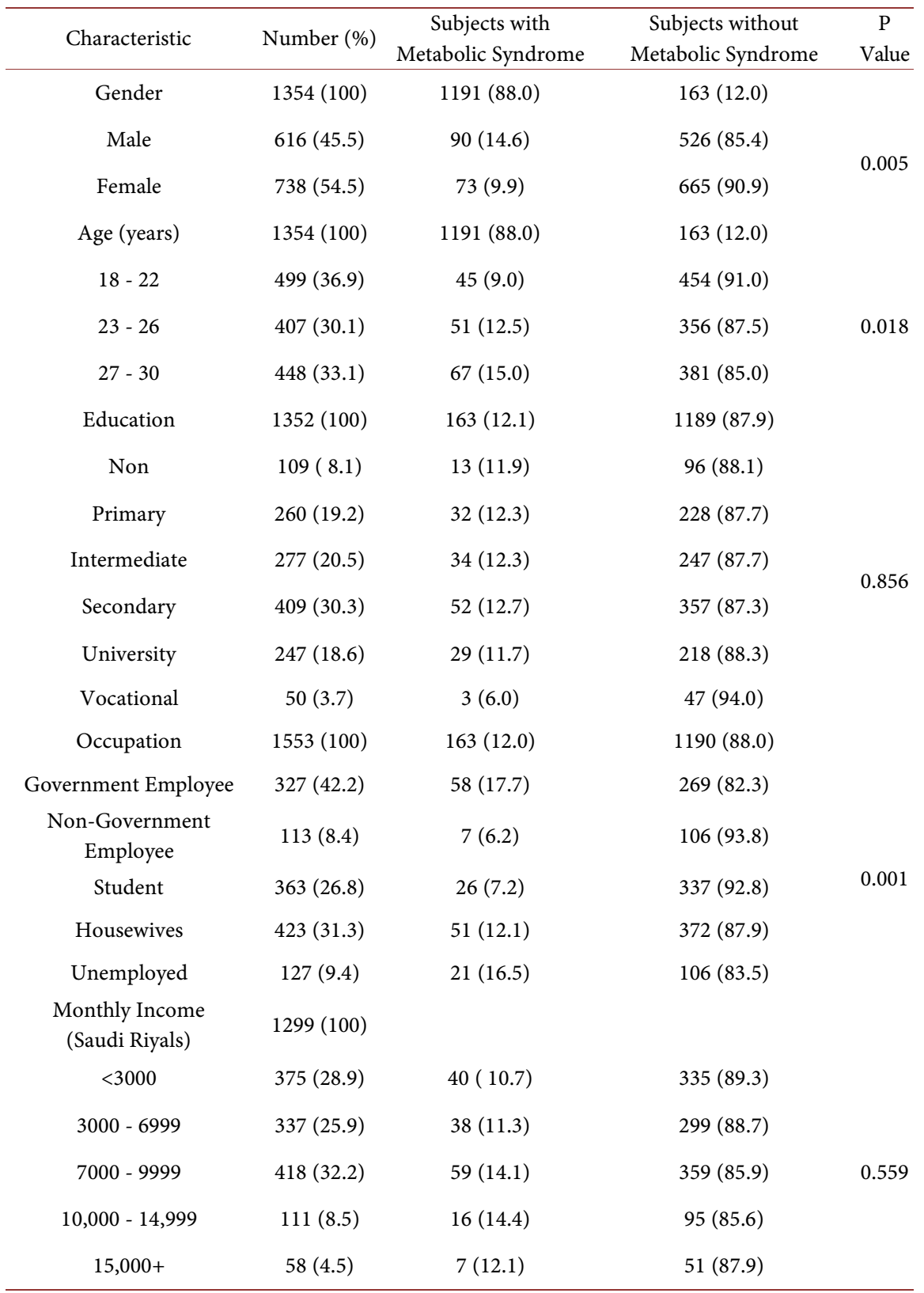

and gender for TGs and physical activity for fasting blood glucose. TGs were significantly more in males $(24.1 \%)$ compared to females (17.3\%) and in subjects 24 years of age with fasting blood glucose significantly higher among subjects with low physical activity level. Other results not depicted in tables revealed no significant differences in all these parameters according to education, occupation, income, smoking status and dietary habits concerning consumption of fruits and vegetables. Table 4 shows result of Logistic regression analysis for significant predictors of MS. All variables entered in bivariate analysis were entered in Logistic Regression Model. Significant predictors included male gender, advancing age, lower level of physical activity and current smoking as shown in Table 5. 
Table 2. Metabolic syndrome according to some life style characteristics.

\begin{tabular}{ccccc}
\hline Characteristic & Number (\%) & $\begin{array}{c}\text { Metabolic } \\
\text { Syndrome } \\
\mathrm{n}(\%)\end{array}$ & $\begin{array}{c}\text { No Metabolic } \\
\text { Syndrome } \\
\mathrm{n}(\%)\end{array}$ & P Value \\
\cline { 2 - 5 } Tobacco Smoking & $1353(100)$ & $1190(88.0)$ & $163(12.0)$ & \\
Current Smokers & $164(12.1)$ & $34(20.7)$ & $130(79.3)$ & \\
Ex-Smoker & $30(2.2)$ & $1(3.3)$ & $29(96.7)$ & 0.001 \\
Never Smoker & $1159(78.5)$ & $128(11.0)$ & $1031(89.0)$ & \\
Physical Activity Level & $1318(100)$ & $1156(87.7)$ & $162(12.3)$ & \\
High & $237(18.0)$ & $19(8.0)$ & $218(92.0)$ & 0.005 \\
Medium & $214(16.2)$ & $18(8.4)$ & $196(91.6)$ & \\
Low & $867(65.8)$ & $125(14.4)$ & $742(85.6)$ & \\
Fruits and Vegetables & $1322(100)$ & $1146(87.7)$ & $161(12.3)$ & \\
Serving per Day & $1259(95.2)$ & $158(12.5)$ & $1101(87.5)$ & 0.189 \\
Below 5 Serving per Day & $63(4.8)$ & $5(7.9)$ & $58(92.1)$ & \\
\hline 5 + Serving per Day & & & & \\
\hline
\end{tabular}

Table 3. Frequency of metabolic syndrome components among the study subject $(\mathrm{n}=$ 1354).

\begin{tabular}{|c|c|c|}
\hline MS Component & Number & Percentage \\
\hline TGs $>1.7 \mathrm{mmol} / \mathrm{L}$ & 276 & 20.4 \\
\hline Fasting Blood Glucose $<5.6 \mathrm{mmol} / \mathrm{L}$ & 214 & 15.8 \\
\hline $\begin{array}{l}\text { Hypertension SBP }>130 \mathrm{mmHg} \text { and/or } \\
\text { DBP }>85 \mathrm{mmHg}\end{array}$ & 267 & 19.7 \\
\hline \multicolumn{3}{|l|}{ WC } \\
\hline Females $>80$ centimeters $(n=738)$ & 326 & 44.2 \\
\hline Male $>94$ centimeters $(n=616)$ & 115 & 18.7 \\
\hline \multicolumn{3}{|l|}{ HDL } \\
\hline Males $<1.03 \mathrm{mmol} / \mathrm{L}(\mathrm{n}=616)$ & 225 & 36.5 \\
\hline Females $<1.29 \mathrm{mmol} / \mathrm{L}(\mathrm{n}=738)$ & 417 & 56.5 \\
\hline
\end{tabular}

\section{Discussion}

About a quarter of the world population is estimated to have MS according to IDF with wide variations according to sociodemographic characteristics among the general population. Prevalence rates of MS among young adults using IDF definition ranged from $1.28 \%$ to over $23.0 \%$ in different communities [10]-[18]. The IDF estimates that $\approx 25 \%$ of the world's population has MS [3] although this estimate varies widely due to the age, ethnicity, and gender of the population studied [4]. Having a slightly raised value of a MS component at a younger age increases the future risk for MS later in life [5]. Therefore, it is important to establish the prevalence of MS components in young adults (18 - 30 years), as the presence of a MS component could represent a lifetime of increased 
Table 4. Parameters of common cutoff points in males and females according to gender, age and physical activity level.

\begin{tabular}{cccc}
\hline Variable/Component & TGs & Elevated Fasting Blood Glucose & High Blood Pressure \\
\hline Gender & & & \\
\hline Males & $24.1 \%$ & 15.1 & 17.4 \\
Females & 17.3 & 16.3 & 19.5 \\
P value & 0.001 & 0.283 & 0.271 \\
\hline Age (years) & & & 18.8 \\
\hline $18-23$ & 17.5 & 14.7 & 16.6 \\
$24-29$ & 23.3 & 15.8 & 28.0 \\
30 - 35 & 20.0 & 21.6 & 0.146 \\
P value & 0.028 & 0.154 & 11.4 \\
\hline Physical Activity Level & & & 14.4 \\
\hline High & 19.4 & 10.5 & 20.3 \\
Medium & 19.1 & 9.7 & 0.054 \\
Low & 21.3 & 18.0 & \\
P value & 0.650 & 0.001 & \\
\hline
\end{tabular}

Table 5. Backward Wald logistic regression analysis for predictors of metabolic syndrome.

\begin{tabular}{|c|c|c|c|c|c|c|c|c|c|}
\hline \multirow[b]{2}{*}{ Predictors } & & \multirow{2}{*}{ B } & \multirow{2}{*}{ S.E. } & \multirow{2}{*}{ Wald } & \multirow{2}{*}{$\mathrm{df}$} & \multirow{2}{*}{ Sig. } & \multirow{2}{*}{$\operatorname{Exp}(B)$} & \multicolumn{2}{|c|}{$\begin{array}{l}\text { 95\% C.I. for } \\
\operatorname{EXP(B)}\end{array}$} \\
\hline & & & & & & & & Lower & Upper \\
\hline \multirow{6}{*}{ Smoking } & & 0.316 & 0.090 & 12.349 & 1 & 0.000 & 1.371 & 1.150 & 1.635 \\
\hline & Physical Activity & 0.000 & .000 & 4.102 & 1 & 0.043 & 1.000 & 1.000 & 1.001 \\
\hline & Total cholesterol & -0.162 & 0.092 & 3.079 & 1 & 0.079 & 0.851 & 0.710 & 1.019 \\
\hline & $\begin{array}{l}\text { Weight for } \\
\text { height ratio }\end{array}$ & -15.625 & 1.341 & 135.763 & 1 & 0.000 & 0.000 & 0.000 & 0.000 \\
\hline & Pulse pressure & -0.040 & .010 & 14.903 & 1 & 0.000 & 0.960 & 0.941 & 0.980 \\
\hline & Constant & 11.740 & 1.089 & 116.157 & 1 & .000 & $125,484.987$ & & \\
\hline
\end{tabular}

a. Variable(s) entered on step 1: c1, age, totsmoke, d1_day, d2_day, T_Activity, bmi, b8, edu_rev, work_rev, inome_rev, WHteatio, pulspressure.

cardiovascular disease risk. Moreover, the early identification of MS components could lead to targeted interventions to prevent the development of the syndrome, and thus reduce cardiovascular disease risk in later life. The results of this study revealed that MS affects $12 \%$ of young adults aged 18 - 30 years. Prevalence rates of MS among young adults using IDF [7] definition ranged from $1.28 \%$ in Romania [10], to $27 \%$ in Russia [11] with prevalence rates in between in many communities. These include prevalence rates of 3.6\% in Turkey [12], $6.8 \%$ among young females in Emirates [13], 6.9\% in Korea [14], 8.7\% in India 
[15], 12.2\% in Brazil [16], 17\% in Canada [17], 21.6\% in USA [14] and $23.1 \%$ in South Africa [18]. Variations in prevalence rates can be expected and explained partly by differences in sociodemographic, geographic and life style characteristics. The risk factors and predictors of MS in this study included male gender, advancing age, smoking, and lower physical activity level. Male gender was a significant predictor of MS among young adults in the majority of studies in other communities [11] [19] [20] [21], Few studies reported that females are more affected than males [10] [12] while a study found no significant difference in MS among young adults according to gender [22]. The inconsistencies can be due to some confounding factors. Smoking and lower physical activity levels were significantly associated with higher prevalence of MS in this study in agreement with many studies worldwide [11] [21] [22] [23] [24]. Education, income and dietary habits were not significantly associated with MS among young Saudi adults in this study in disagreement with studies in other communities [17] [18] [25]. As for the prevalence of the different components of MS in this study lower levels of HDL was the most prevalent component, in both males and females in agreement with many studies worldwide, [10] [17] [19] [20] [22] [24] [26]. This finding raises the possibility that low HDL may be a key marker identifying early pathology associated with the development of MS Prevention of the development of the first MS component may have significant public health benefits as the presence of one component is predictive of the development of MS [6]. An increased waist circumference affected $44 \%$ of females in this study. A study reported that central obesity was the highest component of MS affecting $87.0 \%$ of all the metabolic syndrome subjects [26] [27]. Abdominal obesity was the most common component MS affecting young adults [10] [17] [19]. Significantly more females (51.9\%) presented with increased WC than males $(4.6 \%)$ [11] in accordance with findings of the present study. International studies reported that Atherogenic dyslipidemia defined as low HDL was the most prevalent MS component regardless of the criteria used $(26.9 \%-41.2 \%)$ followed by raised blood pressure (16.6\% - 26.6\%), abdominal obesity $(6.8 \%-23.6 \%)$, atherogenic dyslipidemia defined as raised triglycerides $(8.6 \%-15.6 \%)$, and raised fasting glucose $(2.8 \%-15.4 \%)$ [28].

\section{Strengths and Limitations}

Strengths:

This study, on a large sample of Saudi adults aged 18 - 30 years provides an analysis of correlates and determinants of MS and its individual components by examining the associations and predictors of MS and sociodemographic characteristics and life-style habits. Such information is hoped to be helpful in intervention strategies in this young productive sector of the community.

Limitations:

The study was cross-sectional in design and thus temporal direction of the relationships investigated is unclear. Given that lifestyle behaviors were self-reported, 
it is possible that some responses were influenced by social desirability, subjectivity and recall bias.

\section{Conclusion}

The prevalence of MS is $12 \%$ in young adults. Low HDL is the most prevalent component of MS in young adults and thus may also be the first detectable component of MS in many young adults. Exploring the importance and significance of low HDL in young adults may have considerable public health benefit as interventions aimed at improving low HDL cholesterol levels could reduce future incidence of MS and subsequent clinical disease. Waist circumference in females deserves similar consideration as it's widely prevalent. Early identification of MS components could lead to targeted interventions to prevent the development of the syndrome, and thus reduce cardiovascular disease risk in later life.

\section{Conflicts of Interest}

The author declares no conflicts of interest regarding the publication of this paper.

\section{References}

[1] Alberti, K.G., Eckel, R.H., Grundy, S.M., et al. (2009) Harmonizing the Metabolic Syndrome: A Joint Interim Statement of the International Diabetes Federation Task Force on Epidemiology and Prevention. National Heart, Lung, and Blood Institute, American Heart Association, World Heart Federation, International Atherosclerosis Society; and International Association for the Study of Obesity. Circulation, 120, 1640-1645. https://doi.org/10.1161/CIRCULATIONAHA.109.192644

[2] Aljohani, N. (2014) Metabolic Syndrome: Risk Factors among Adults in Kingdom of Saudi Arabia. Journal of Family and Community Medicine, 21, 170-175. https://doi.org/10.4103/2230-8229.142971

[3] O’Neill, S. and O'Driscoll, L. (2015) Metabolic Syndrome: A Closer Look at the Growing Epidemic and Its Associated Pathologies. Obesity Reviews, 16, 1-12. https://doi.org/10.1111/obr.12229

[4] Kaur, J. (2014) A Comprehensive Review on Metabolic Syndrome. Cardiology Research and Practice, 2014, Article ID: 943162. https://doi.org/10.1155/2014/943162

[5] Gündogan, K., Bayram, F., Capak, M., et al. (2009) Prevalence of Metabolic Syndrome in the Mediterranean Region of Turkey: Evaluation of Hypertension, Diabetes Mellitus, Obesity, and Dyslipidemia. Metabolic Syndrome and Related Disorders, 7, 427-434. https://doi.org/10.1089/met.2008.0068

[6] Cheung, B.M.Y., Wat, N.M.S., Tam, S., et al. (2008) Components of the Metabolic Syndrome Predictive of Its Development: A 6-Year Longitudinal Study in Hong Kong Chinese. Clinical Endocrinology, 68, 730-737. https://doi.org/10.1111/j.1365-2265.2007.03110.x

[7] Kassi, E., Pervanidou, P., Kaltsas, G. and Chrousos, G. (2011) Metabolic Syndrome: Definitions and Controversies. BMC Medicine, 9, 48. https://doi.org/10.1186/1741-7015-9-48

[8] WHO, EMRO (2005) STEPwise WHO. 
http://www.who.int/chp/steps/2005_SaudiArabia_STEPS_Report_EN.pdf

[9] Bonita, R., de Courten, M., Dwyer, T., Jamorzik, K. and Winkelmann, R. (2001) Surveillance of Risk Factors for Non Communicable Diseases. The WHO Stepwise Approach, WHO.

[10] Nitescu, M., et al. (2012) Prevalence of Metabolic Syndrome Parameters in a Romanian Population of Young Adults. Revista Română de Medicină de Laborator, 20, 207-214.

[11] Ragino, Y., Kovalkova, N., Travnikova, N., Denisova, D. and Voevoda, M. (2017) Prevalence of Metabolic Syndrome in Young Adults in Russia. Atherosclerosis, 263, e172.

[12] Soysal, A., et al. (2005) The Prevalence of Metabolic Syndrome among Young Adults in Emir, Turkey. Anadolu Kardiyoloji Dergisi, 5, 196-201.

[13] Al Dhaheri, A.S., Mohamad, M.N., Jarrar, A.H., Ohuma, E.O., Ismail, L.C., Al Meqbaali, F.T., et al. (2016) A Cross-Sectional Study of the Prevalence of Metabolic Syndrome among Young Female Emirati Adults. PLoS ONE, 11, e0159378.

[14] Park, J., et al. (2008) A Comparison of the Prevalence of the Metabolic Syndrome in the United States (US) and Korea in Young Adults Aged 20 to 39 Years. Asia Pacific Journal of Clinical Nutrition, 17, 471-482.

[15] Manjunath, D., Uthappa, C.K., Kattula, S.R., Allam, R.R., Chava, N. and Oruganti, G. (2014) Metabolic Syndrome among Urban Indian Young Adults: Prevalence and Associated Risk Factors. Metabolic Syndrome and Related Disorders, 12, 381-389. https://doi.org/10.1089/met.2014.0003

[16] Carvalho, C.A., Silva, A.A.M., Assunção, M.C.F., Fonseca, P.C.A., Barbieri, M.A., Bettiol, H., Shivappa, N. and Hébert, J.R. (2018) The Dietary Inflammatory Index and Insulin Resistance or Metabolic Syndrome in Young Adults. Nutrition, 58, 187-193. https://doi.org/10.1016/j.nut.2018.07.014

[17] Riediger, N.D. and Clara, I. (2011) Prevalence of Metabolic Syndrome in the Canadian Adult Population. CMAJ, 183, E1127-E1134.

[18] Sekgala, M.D., Mchiza, Z.J., Parker, W.A. and Monyeki, K.D. (2018) Dietary Fiber Intake and Metabolic Syndrome Risk Factors among Young South African Adults. Nutrients, 10, E504. https://doi.org/10.3390/nu10040504

[19] Martínez-Torres, J., et al. (2017) A Cross-Sectional Study of the Prevalence of Metabolic Syndrome and Associated Factors in Colombian Collegiate Students: The Fuprecol-Adults Study. International Journal of Environmental Research and Public Health, 14, 233. https://doi.org/10.3390/ijerph14030233

[20] Sabir, F.M., Hassan, D.A. and Elamin, M.I. (2016) Prevalence of Metabolic Syndrome among Young Sudanese University Students Using Three Different Criteria of WHO, IDF and NCEP-ATP III. Pediatrics and Neonatal Nursing Journal, 2.

[21] Buckland, G., Salas-Salvador, J., Roure, E., Bulló, M. and Serra-Majem, L. (2008) Sociodemographic Risk Factors Associated with Metabolic Syndrome in a Mediterranean Population. Public Health Nutrition, 11, 1372-1378. https://doi.org/10.1017/S1368980008003492

[22] Damiri, B., Aghbar, A., Alkhadour, S. and Arafat, Y. (2017) Characterization and Prevalence of Metabolic Syndrome among Overweight and Obese Young Palestinian Students at An-Najah National University. Diabetes \& Metabolic Syndrome Clinical Research \& Reviews, 12, 343-348.

[23] Kongara, S., Arepalli, S.K., et al. (2017) A Study on the Prevalence of Obesity and Metabolic Syndrome among Students of a Medical College. International Journal of 
Research in Medical Sciences, 5, 2331-2337.

https://doi.org/10.18203/2320-6012.ijrms20172012

[24] Yahia, N., Brown, C.A., Snyder, E., Cumper, S., Langolf, A., Trayer, C. and Green, C. (2017) Prevalence of Metabolic Syndrome and Its Individual Components among Midwestern University Students. Journal of Community Health, 42, 674-687. https://doi.org/10.1007/s10900-016-0304-5

[25] Pérez-Martínez, P., Mikhailidis, D.P., Athyros, V.G., Bullo, M., Couture, P., Esposito, K., Fitó, M., Garaulet, M., Giugliano, D., et al. (2017) Lifestyle Recommendations for the Prevention and Management of Metabolic Syndrome: An International Panel Recommendation Nutrition Reviews, 75, 307-326. https://doi.org/10.1093/nutrit/nux014

[26] Usha, S.M.R., Chandrika, N., Shetty, H.V. and Reena, R. (2014) A Study of the Components of Metabolic Syndrome in Young Adults. Biomedical Research, 25, 45-50.

[27] Adam, F.M.S., Diatri, M.C., Adam, J.M.F., Seweng, A. and Tai, E.S. (2015) Prevalence of Metabolic Syndrome in Young Adults. International Journal of Science and Research, 4, 1320-1323.

[28] Nolan, P.B., Carrick-Ranson, G., Stinear, J.W., Reading, S.A. and Dalleck, L.C. (2017) Prevalence of Metabolic Syndrome and Metabolic Syndrome Components in Young Adults: A Pooled Analysis. Preventive Medicine Reports, 7, 211-215. https://doi.org/10.1016/j.pmedr.2017.07.004 